\title{
A NEW CLASS OF HYPERFINITE KADISON-SINGER FACTORS
}

\author{
FEI MA AND YE ZHANG
}

Abstract. In this paper, we construct a new class of hyperfinite Kadison-Singer factors on separable Hilbert spaces, and we show that each of these Kadison-Singer factors is isomorphic to a subalgebra of CSL algebra. Moreover, a sufficient and necessary condition for two of these Kadison-Singer factors being isometrically isomorphic is given. Finally, we obtain that every norm preserving automorphism on these Kadison-Singer algebras is inner. Mathematics subject classification (2010): 47L35, 47L75.

Keywords and phrases: Kadison-Singer algebra, Kadison-Singer lattice, hyperfinite KS-factor, CSL algebra.

\section{REFERENCES}

[1] A. J. Dong, C. J. Hou, On some automorphisms of a class of Kadison-Singer algebras, Linear Algebra Appl. 436 (2012) 2037-2053.

[2] A. J. Dong, C. J. Hou, J. TAN, Classification of Kadison-Singer lattices in matrix algebras, Acta Math. Sin., Chin. Series, 54 (2011) 333-342.

[3] A. J. Dong, D. WANG, On strong Kadison-Singer algebras, Acta Math. Sinica (Englishi), 29 (2013) 2219-2232.

[4] C. J. Hou, Cohomology of a class of Kadison-Singer algebras, Sci. China Math. 53 (2010) $1827-$ 1839.

[5] C. J. Hou, Derivations of a class of Kadison-Singer algebras, Linear Algebra Appl. 466 (2015) 241253.

[6] C. Hou, W. Yuan, Minimal generating reflexive lattices of projections in finite von Neumann algebras, Math. Ann. 353 (2012) 499-517.

[7] J. R. Ringrose, Super-diagonal forms for compact linear operators, Proc. Lond. Math. Soc. 12 (1962) 367-384.

[8] J. R. Ringrose, On some algebras of operators, Proc. Lond. Math. Soc. 15 (1965) 61-83.

[9] L. G. WANG, W. YUAN, A new class of Kadison-Singer algebras, Expositiones Math. 29 (2011) $126-132$.

[10] L. M. GE, W. YuAn, Kadison-Singer Algebras, I-Hyperfinite Case, Proc. Natl. Acad. Sci. USA, 107 (2010) 1838-1843.

[11] L. M. Ge, W. YuAn, Kadison-Singer Algebras, II -Finite Case, Proc. Natl. Acad. Sci. USA, 107 (2010) 4840-4844.

[12] R. KADISON, I. Singer, Triangular operator algebras. Fundamentals and hyperreducible theory, Amer J. Math. 82 (1960) 227-259.

[13] R. Kadison, J. Ringrose, Fundamentals Of The Theory Of Operator Algebras, volume I and II, Academic Press, Orlando, 1983 and 1986.

[14] R. POWERs, Representations of uniformly hyperfinite algebras and their associated von Neumann rings, Ann.Math., 86:138-171, 1967.

[15] W. M. WU, W. YUAN, On generators of abelian Kadison-Singer algebras in matrix algebras, Linear Algebra Appl. 440 (2014) 197-205.

[16] W. YuAn, Kadison-Singer algebras, Doctoral Thesis (University of New Hampshire, NH), 2009.

[17] Y. H. REN, W. M. Wu, Some new classes of Kadison-Singer lattices in Hilbert spaces, Science China (Mathematics) 57 (2014) 837-846. 